\title{
Epstein-Barr virus recruits PDL1-positive cells at the microenvironment in pediatric Hodgkin lymphoma
}

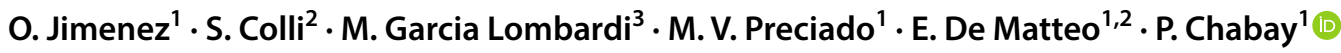

Received: 4 June 2020 / Accepted: 28 October 2020

(c) Springer-Verlag GmbH Germany, part of Springer Nature 2020

\begin{abstract}
Background Classic Hodgkin lymphoma (cHL) is a lymphoid malignancy in which the microenvironment, where the neoplastic cells are immersed, contributes to the lymphomagenesis process. Epstein-Barr virus (EBV) presence also influences cHL microenvironment composition and contributes to pathogenesis. An increase in PDL1 expression in tumor cells and at the microenvironment was demonstrated in adult cHL. Therefore, our aim was to assess PD1/PDL1 pathway and EBV influence on this pathway in pediatric cHL, given that in Argentina, our group proved a higher incidence of EBV-associated pediatric lymphoma in children.

Methods For that purpose, EBV presence was assessed by in situ hybridization, whereas PD1 and PDL1 expressions were studied by immunohistochemistry. PDL1 genetic alterations were analyzed by FISH, and survival was evaluated for PD1 and PDL1 expressions.

Results EBV presence demonstrated no influence neither on PD1 expression at the microenvironment nor on PDL1 expression at HRS tumor cells. Unexpectedly, only $38 \%$ pediatric cHL displayed PDL1 genetic alterations by FISH, and no difference was observed regarding EBV presence. However, in EBV-related cHL cases, a higher number of PDL1 + cells were detected at the microenvironment.

Conclusion Even though a high cytotoxic environment was previously described in EBV-related pediatric cHL, it might be counterbalanced by an immunoregulatory micro-environmental PDL1 + niche. This regulation may render a cytotoxic milieu that unsuccessfully try to eliminate EBV + Hodgkin Reed Sternberg tumor cells in pediatric patients.
\end{abstract}

Keywords Epstein-Barr virus · Pediatric Hodgkin lymphoma · Microenvironment · PDL1 · PD1

Electronic supplementary material The online version of this article (https://doi.org/10.1007/s00262-020-02787-2) contains supplementary material, which is available to authorized users.

P. Chabay

paola_chabay@yahoo.com.ar

1 Molecular Biology Laboratory, Pathology Divison, Multidisciplinary Institute for Investigation in Pediatric Pathologies (IMIPP), CONICET-GCBA, Hospital de Niños R. Gutiérrez, Gallo 1330, C1425EFD Buenos Aires, Argentina

2 Pathology Division, Ricardo Gutiérrez Children's Hospital, Buenos Aires, Argentina

3 Oncology Division, Ricardo Gutiérrez Children's Hospital, Buenos Aires, Argentina

\section{Introduction}

Hodgkin lymphoma (HL) is a lymphoid malignancy that primarily involves lymph nodes, but possesses a distinctive morphological presentation with a minority of neoplastic cells, namely Hodgkin with their variants Reed-Sternberg (HRS) cells, which generally comprise less than $1 \%$ of the total cell population, immerse in a large majority of nonmalignant reactive immune cells [1]. Those infiltrating cells attracted because of HRS cells secretion of chemokines and cytokines, are beneficial for the tumor [2].

Evading immune response is a recognized hallmark of cancer [3]. Cytotoxic T cells (CTLs) are responsible for killing tumor and virus-infected cells, and for controlling persistent viral infections. Nevertheless, persistent antigenic stimulation leads to CD8 + T cell exhaustion, characterized by the induction of a hypo-proliferative state, the loss of the ability to produce cytokines IL2, TNF $\alpha$ and IFN $\gamma$, and 
granzyme B (GrB), whereas inhibitory cytokines derived from regulatory T cells (Tregs), such as IL10 and transforming growth factor $\beta$ (TGF $\beta$ ), also suppress the function of effector $\mathrm{T}$ cells $[4,5]$. Exhausted $\mathrm{T}$ cell loses the ability to eliminate neoplastic cells; therefore, this process plays an important role in the development of cancer, including hematologic malignancies [6]. One of the pathways of immune exhaustion involves the PD1 molecule and its ligand PDL1 [7]. PD1 is expressed by tumor-infiltrating lymphocytes (TILs) in the microenvironment in several hematologic malignancies including follicular lymphoma (FL), diffuse large B cell lymphoma (DLBCL), and cHL [8-10]. On the other hand, PDL1 could be upregulated in HRS cells, driving to the expression of PD1 in T cells at the microenvironment [11]. This microenvironment is a niche for HRS cells positive for PDL1, which could also be expressed by tumor-associated macrophages (TAM) [12]. Binding of PD1 to its ligand PDL1 inhibits cytokine production and cellcycle progression in $\mathrm{T}$ cells, and represents an important checkpoint in the regulation of immune responses [13], and represents the key to the development of monoclonal antibodies directed against these control points in the treatment of several tumors [7].

PDL1 is encoded on the short arm of chromosome 9 (9p24.1), close to the locus of PDL2 and Jak2. It has been demonstrated that overexpression of the PDL1 is consequence of chromosome 9p24.1 alterations (56\% show copy gain; 5\% polysomy and 36\% amplification) observed in most cHL adult cases [12]. The interaction of PD1 with PDL1 occurs between PD1 + TILs and PDL1 + lymphoma cells, but also between PD1 + TILs and PDL1 + non-malignant cells at the microenvironment [13]. In cHL, PDL1 + cells expressed by TAMs physically co-localize with HRS cells in a micro-environmental niche, and HRS cells are enriched for contacts with $\mathrm{T}$ cells expressing CTLA4 in a higher frequency than PD1 or LAG3 [14, 15].

According to previous reports, the increase in PDL1 expression is associated with a poor survival, so it is usually considered as a prognostic biomarker [12]. The increase in PDL1 expression in tumor cells as well as at the microenvironment was associated to Epstein-Barr virus (EBV) presence in DLBCL [16]. In fact, EBV infection was proposed as an alternative mechanism for PDL1 induction given that 9p24.1 amplification and EBV infection were mutually exclusive in a series of cHLs; furthermore, LMP1 viral latent protein increases PDL1 promoter activity in vitro via JAK3 [17], enlarging to EBV-associated neoplasias the spectrum of tumor susceptible to PD1 blockade. However, some reports argue against this finding, given that the distribution of PDL1 genetic alterations in EBV + and EBV - was equivalent in an adult cHL series [12]. Furthermore, PDL1 gene abnormalities were higher in EBV-positive T cell and NK-cell lymphomas, as well as EBV-associated DLBCL
[18]. In contrast, only $11 \%$ of EBV + gastric carcinoma displays PDL1 amplification [19].

EBV is a gammaherpesvirus with growth-transforming properties in vitro on B cells, the most important target cell, which is why it was also linked to several B cell lymphomas, like Burkitt's lymphoma, DLBCL and HL. In developed countries, EBV is detected in $30-35 \%$ of cHL cases, whereas this rate is significantly increased in developing populations, where mixed cellularity (MC) subtype is prevalent $[20,21]$. In Argentina, the overall EBV-association rate is $59 \%$ in pediatric cHL, but even though MC is still the prevalent subtype, nodular sclerosis subtype prevalence is rising in the last years [22]. Nevertheless, EBV positivity is statistically increased in children younger than 10 years in our country [23]. EBV-associated cHL exhibits Latency II pattern, in which EBERs transcripts as well as LMP1 and LMP2A latent viral proteins are expressed by HRS cells. Even though the role of EBV in cHL pathogenesis is not yet entirely disclosed, latency II antigens are involved in the rescue of germinal center (GC) B cells with crippling mutations from apoptosis to become an HRS cell [20].

In line with this, EBV presence also has influence on $\mathrm{cHL}$ microenvironment composition, to contribute to lymphomagenesis process. In EBV-associated cHL in adult patients, a higher number of cytotoxic CD8 $+\mathrm{T}$ cells, along with regulatory $\mathrm{CD} 4+\mathrm{T}$ cells (Tregs) that secrete IL10, were demonstrated [24]. In pediatric cHL, EBV + cases exhibit a cytotoxic/Th1 profile, with an antitumoral M1 macrophage polarization pattern $[25,26]$. In line with this, our group proved the prevalence of a cytotoxic and M1 polarization pattern in pediatric cHL from Argentina [27], which is apparently unable to eliminate EBV + HRS cells. This finding prompted us to hypothesize that there is an immune regulatory mechanism counteracting those antitumoral responses at the microenvironment. Therefore, our aim was to characterize PD1/PDL1 pathway in HRS cells microenvironment to disclose its involvement in the pathogenesis of pediatric EBV-associated cHL.

\section{Methods}

\section{Patients and samples}

Formalin-fixed paraffin-embedded tissue biopsies (FFPE) of 80 patients were analyzed, taking into account the availability of sufficient material from the archives of the Pathology Division of the Ricardo Gutiérrez Children's Hospital, Buenos Aires, Argentina. The age range was 2-18 years (median: 9.5 years). This series enlarges the data published previously [27]. Institutional guidelines regarding human experimentation were followed, and they were in accordance to the Helsinki Declaration of 1975. The Ethical Committee 
of the institution approved the study, and all the patients' guardians gave informed consent for the study. Diagnosis was made from biopsies taken from the primary tumor and cases were classified by the pathologist (E.D.M) by immunohistochemical staining according to the updated World Health Organization (WHO) scheme for lymphomas [28]. From each case, representative tumor areas rich in HRS selected by pathologists of the service were selected.

\section{EBERs in situ hybridization (ISH)}

In situ hybridization for EBERS was performed in FFPE tissue sections using labeled oligonucleotides with fluorescein isothiocyanate (FITC) as probes (DAKO) according to the manufacturer's instructions. An anti-FITC monoclonal antibody labeled with alkaline phosphatase was used to detect hybridized sites. An EBV-positive post-transplant lymphoproliferative disorder was used as a positive control.

\section{Immunohistochemistry (IHC)}

On the FFPE biopsies (3-4 $\mu \mathrm{m})$, immunohistochemistry was performed with the panel of antibodies for: PD1: CD279 (clone NAT 105, dil: 1/50, Abcam), PDL1: CD 274 (clone 4E54, Abcam), IL10 (Ab34843, Abcam), TGF $\beta$ (Ab9758, Abcam). Antigenic recovery was performed using Tris-EDTA buffer $\mathrm{pH}=9.0$ for the detection of PDL1 and TGF $\beta$, and for PD1 and IL10 with sodium citrate buffer $\mathrm{pH}=6.0$. After incubation with the primary antibody, immunodetection was performed using the Vectastain-ABC-Peroxidase kit, using diaminobenzidine (DAB) as a chromogen. As a positive control for each marker, a tonsil tissue was selected, observing a brown pattern in membrane and cytoplasm in positive cells. The analysis of cell populations was performed by light field microscopy (Carl Zeiss, Scope.A1). The results were expressed as positive cells per area unit (cells $+/ \mathrm{mm}^{2}$ ).

Immunostaining was used to assess viral LMP1 expression in tumor cells on EBV + (by EBERs ISH) tissue samples, using monoclonal antibodies CS1-4 (Dako). Antigenic recovery was performed with sodium citrate buffer $\mathrm{pH}=6.0$. IHC detection of primary antibody was carried out using a universal streptavidin-biotin-complex peroxidase detection system (UltraTek HRP Anti-Polyvalent Lab Pack, ScyTek) according to the manufacturer's instructions. An EBV-positive post-transplant lymphoproliferative disorder was used as a positive control.

\section{Fluorescent in situ hybridization (FISH)}

In a subgroup of 37 patients FISH for the PDL1 gene was performed, based on the quality of FFPE samples. FFPE biopsies were hydrated with xilol and ethanol, pretreated with heat and pepsin for $15 \mathrm{~min}$, and then incubated with specific probes that hybridize with the PDL1 gene in the 9p24.1 region of double-color design, labeled with red fluorochrome, and control probe in the pericentromeric region of chromosome 9 (CEP 9) labeled with green fluorochrome (Lexel Laboratories). Prior to the addition of the probe, the tissues were treated with a solution of pepsin $(0.005 \%$ pep$\sin )$ at $37^{\circ} \mathrm{C}$. Subsequently, two washes were performed with PBS1X for $5 \mathrm{~min}$ and dried at room temperature. Then, a dehydration step was carried out with ethanol solution in increasing order of ethanol concentration $(70 \%, 90 \%$ and $100 \%$ for $3 \mathrm{~min}$ ). The slides were hybridized according to the manufacturer's instructions. DAPI was used for nuclear staining. Approximately 50 HRS cells per case were analyzed. Taking into account the ratio of PDL1/CEP9 signals, three conditions were defined: amplification (PDL1/ CEP9 $\geq 3: 1$ ), PDL1 copy gain (PDL1/CEP9 $>1: 1$ but $<3: 1$ ) and polysomy of chromosome 9 (PDL1/CEP9 1: 1 but with $>$ two copies of each probe).

\section{Statistical analysis}

Statistical analysis was performed using GraphPad Prism 5 (GraphPad Software Inc, San Diego California USA). Categorical variables were analyzed using Fisher's exact test or Chi-Square test when necessary. Mann-Whitney test was used to compare the means between groups in relation to EBV presence. Correlations between data were determined using Spearman or Pearson rank correlation index, when appropriate. All tests were two-tailed, and $p<0.05$ was considered statistically significant. The follow-up time was defined as the length of time from the date of diagnosis to either last follow-up date or when a given event occurs. Event-free survival (EFS) was measured from the date of diagnosis and first treatment to either date of disease progression or discontinuation of treatment for any reason or censoring date when patient's loss of contact or withdrawal from the study. For survival analyses related to reactive microenvironment expression, two groups were considered: the group of patients with expression values above the 50th percentile (median) versus the group below this point. Kaplan-Meier curves based on the abovementioned median thresholds were generated and statistical significance of each marker was determined using the log-rank test.

\section{Results}

Concerning histological characterization, as expected for a developing population, MC subtype was proved in 56\% of cases (39/69 with histological subtype data), whereas nodular sclerosis subtype was characterized in 39\% (27/69). The remaining cases were lymphocyte depletion, 
lymphocyte-rich or not known ( $n=11)$ (Suppl Table 1). EBV expression was proved in $69 \%$ of patients $(55 / 80)$ by means of EBERs ISH, and LMP1 expression by IHC was observed in all 55 EBER-positive cases (Fig. 1a, b). As previously described in our previous work [23], represented in part by this series, both MC subtype and age under 10 years old were statistically associated to EBV + cases ( $p<0.05$, Fisher's exact test). Based on those results, two analysis patterns were used for evaluation of PD1/PDL1 pathway parameters: $\mathrm{EBV}+$ and EBV - cases, as well as patients younger and older than 10 years old.

Upregulation of PD1 expression on tumor-infiltrating lymphocytes in different types of cancers leads to an exhausted phenotype [5]. Furthermore, this upregulation was characterized in models of chronic viral infection [11]. Therefore, PD1 cell count was compared in EBV-associated versus EBV-non-associated pediatric cHL, but no difference was observed in PD1 + cell count between EBV + and EBV - cases ( $p>0.05$, Mann-Whitney test) (Fig. 1c). In addition, PD1 expression was compared between children younger and older than 10 years old, since we observed age differences in pediatric cHL cases [27]. However, no significant differences were found in PD1 + cell numbers between both age groups ( $p>0.05$, Mann-Whitney test) (Fig. 2). IL10 and TGF $\beta$ are inhibitory cytokines that suppress the function of effector T cells [4]. To confirm if those cytokines could drive $\mathrm{T}$ cells into an exhausted phenotype, PD1 expression was correlated with each of them in a subset of 43 pediatric cHL patients. Median IL10 and TGF $\beta$-positive cell counts in relation to EBV presence and age groups are detailed in supplementary Table 2, while survival analysis with both cytokine expression is presented in supplementary Fig. 1. Neither IL10 nor TGF $\beta$ is statistically associated with survival ( $p>0.05, \log$-rank test). In the whole series, neither IL10 nor TGF $\beta$ displayed a significant correlation with PD1 + cell numbers $(p>0.05$, Spearman correlation test). However, in EBV + cases, PD1 + cells showed a significant positive correlation with the IL10 + ones ( $r=0.4257$, $p=0.0239$, Spearman correlation test). In line with this, in patients younger than 10 years old, most EBV-associated cases, a statistical positive correlation was demonstrated between IL10 + and PD1 + cells $(r=0.5866, p=0.0065$, Spearman correlation test). In contrast, TGF $\beta+$ cells only revealed a positive correlation with the PD1 + ones in EBV - cases $(r=0.8434, p=0.0085$, Spearman correlation test).

PDL1, as the ligand of PD1, can be expressed in malignant HRS and in non-malignant cells at the microenvironment, as described for DLBCL, to evade host immune response. It was described that EBV presence may influence
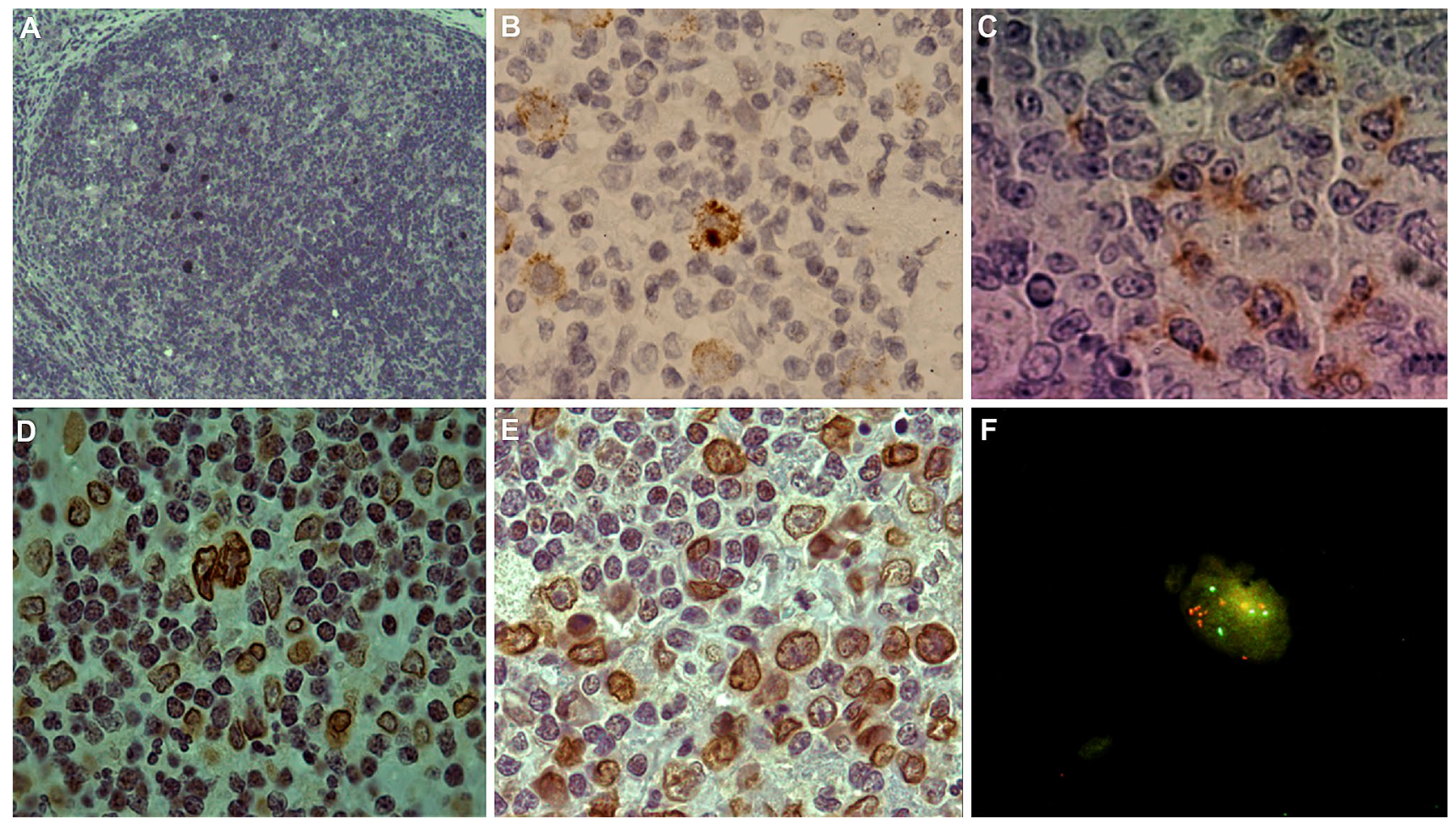

Fig. 1 Representative staining undertaken on sections from cHL biopsies. Nuclear localization of the EBERs in neoplastic cells by ISH (a). Membranous and cytoplasmic localization of LMP1 in neoplastic cells by IHC (b). Membranous and cytoplasmic staining of
PD1 in the microenvironment by IHC (c). Membranous and cytoplasmic localization of PDL1 in neoplastic cells (d) and in the microenvironment (d and e). Amplification of PDL1 gene in the nucleus of tumor cells by FISH (f) 
Fig. 2 Comparison of mean cell count of immune cell markers PD1, PDL1in HRS cells, and PDL1 at the microenvironment between $\mathrm{EBV}+(n=55)$ and EBV - $(n=25)$ cases (a) and between children younger or equal $(n=41)$, and older $(n=30)$ than 10 years $(\mathbf{b})$. Black bars indicate EBV + cases and $\leq 10$ years, and grey bars indicate EBV - and $>10$ years. Error bars indicate SD. The $p$-value is from the Mann Whitney test
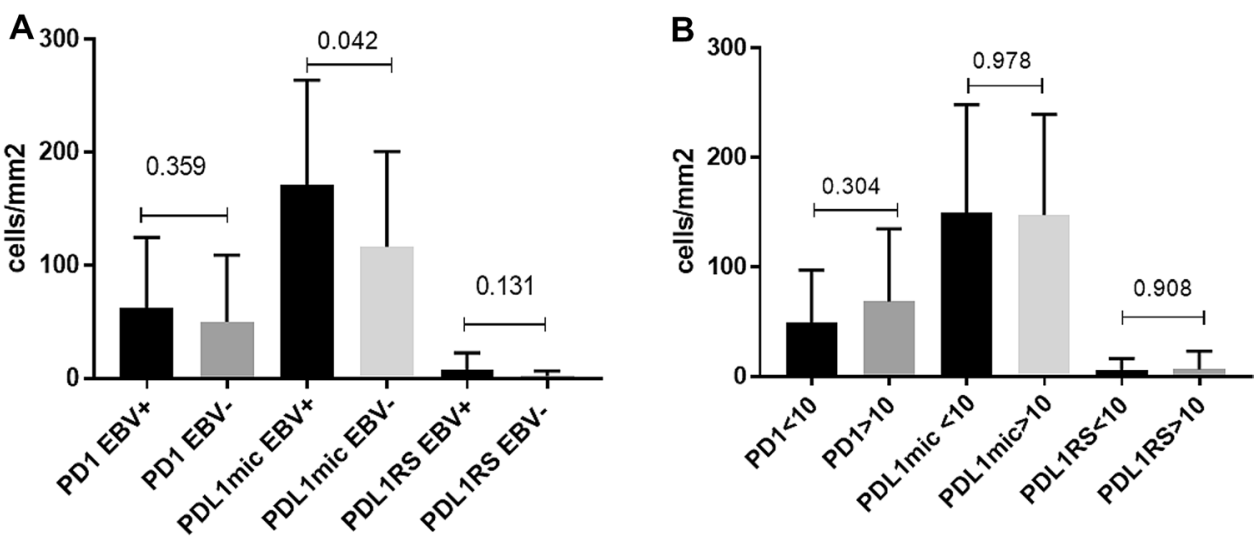

its expression [16]. PDL1 presence in HRS cells by means of IHC was observed in $44 \%$ (35/80) pediatric cHL cases (Fig. 1d), 77\% (27/35) were EBV + cases, while in the remaining 45 cases in which PDL1 expression was not observed, EBV positivity was proved in 69\% (31) of cases, without statistical differences ( $p>0.05$, Fisher's exact test). In line with this, when PDL1 + HRS cells were counted, no differences were found between EBV-associated and nonassociated cases ( $p>0.05$, Mann-Whitney test).

The influence of EBV presence in PDL1 genetic alterations in tumor cells is still under discussion. To disclose viral influence on a pediatric population with increased EBVassociated cHL prevalence, 9p24.1 genetic alterations were studied in a subgroup of 37 patients with good-quality samples by means of FISH. Amplification of PDL1 gene (PDL1/ CEP9 $\geq 3$ ) in HRS cells was observed in 8\% (3/37) of cases, PDL1 copy gain (PDL1/CEP9 $>1: 1$ but $<3: 1$ ) was proved in $16 \%$ (6/37) of cases, whereas in $14 \%(5 / 37)$ of cases, HRS cells showed cells with PDL1 amplification and cells with PDL1 copy gain (Fig. 1f). In the remaining 62\% (23/37) of cases, no 9 p24.1 genetic alterations were observed. Sixty-five percent (24/37) of cases were EBV + (10 cases with PDL1 rearrangement), while the remaining cases were EBV - (5 cases with PDL1 rearrangement). Neither PDL1 amplification nor copy gain was statistically associated to EBV presence ( $p>0.05$, Fisher's exact test). Furthermore, when PDL1 + mean cell count was compared between cases with and without genetic alterations, no statistical differences were demonstrated ( $p>0.05$, Mann-Whitney test).

Microenvironment plays a key role in the pathogenesis of cHL, and PDL1 plays an important role, given that PDL1 + cells co-localize with HRS cells in a micro-environmental niche [14]. In pediatric $\mathrm{cHL}$, age and EBV presence influence microenvironment composition [25, 27]. Therefore, specific PDL1 expression at the microenvironment in our series was analyzed in relation to EBV-positive and age (Fig. 1e). No differences in the mean of PDL1 cell count were proved when comparing patients younger vs. older than 10 years old ( $p>0.05$, Mann-Whitney test). Remarkably, in
$\mathrm{EBV}+$ cases, PDL1 + cell count was statistically higher than in the EBV - ones ( $p=0.042$, Mann-Whitney test) (Fig. 2).

In adult cHL, PDL1 expression and genetic alterations were associated with survival [12]. In our series, the PD1 and PDL1 median cell numbers at the microenvironment were used as cutoff value for the survival analysis, whereas concerning PDL1 expression in HRS cells, positive versus negative cases were applied as cutoff. Unexpectedly, neither PD1 nor PDL1 expressions in HRS cells or at the microenvironment were associated to survival in pediatric $\mathrm{cHL}$ patients ( $p>0.05$, log-rank test) (Fig. 3).

\section{Discussion}

In the last years, the PD1-PDL1 pathway has been extensively studied as a target for immunotherapy approaches, since blocking this interaction triggers antitumor responses and better outcome. PD1 is upregulated on T cells upon activation and it remains highly expressed on exhausted T cells, reason why is expressed on tumor-infiltrating lymphocytes. Meanwhile, PDL1 is upregulated on tumor cells, as well as in macrophages and dendritic cells at the tumor microenvironment [29], for such reason, it is evaluated in both scenarios [16]. This pathway was studied in several types of adult lymphomas.

In DLBCL, PDL1 expression in tumor cells was associated with shorter survival [16, 30, 31], whereas no significant differences were observed in PDL1 expression at the microenvironment when cases with PDL1 + expression were compared versus the PDL1- ones [16]. Wang et al. reported only a $10 \%$ of DLBCL cases with PDL1 gene alterations, and, surprisingly, the cases with 9p24.1 amplification had a trend of better event-free survival [32]. In adult cHL, amplification and copy gain of 9p24.1 PDL1 gene was proved in $36 \%$ and $56 \%$ of cases, and it was associated with shorter survival [12]. Regarding PDL1 expression at the microenvironment, higher PDL1 expression was proved around HRS cells, in micro-environmental niche [14]. Only one group 

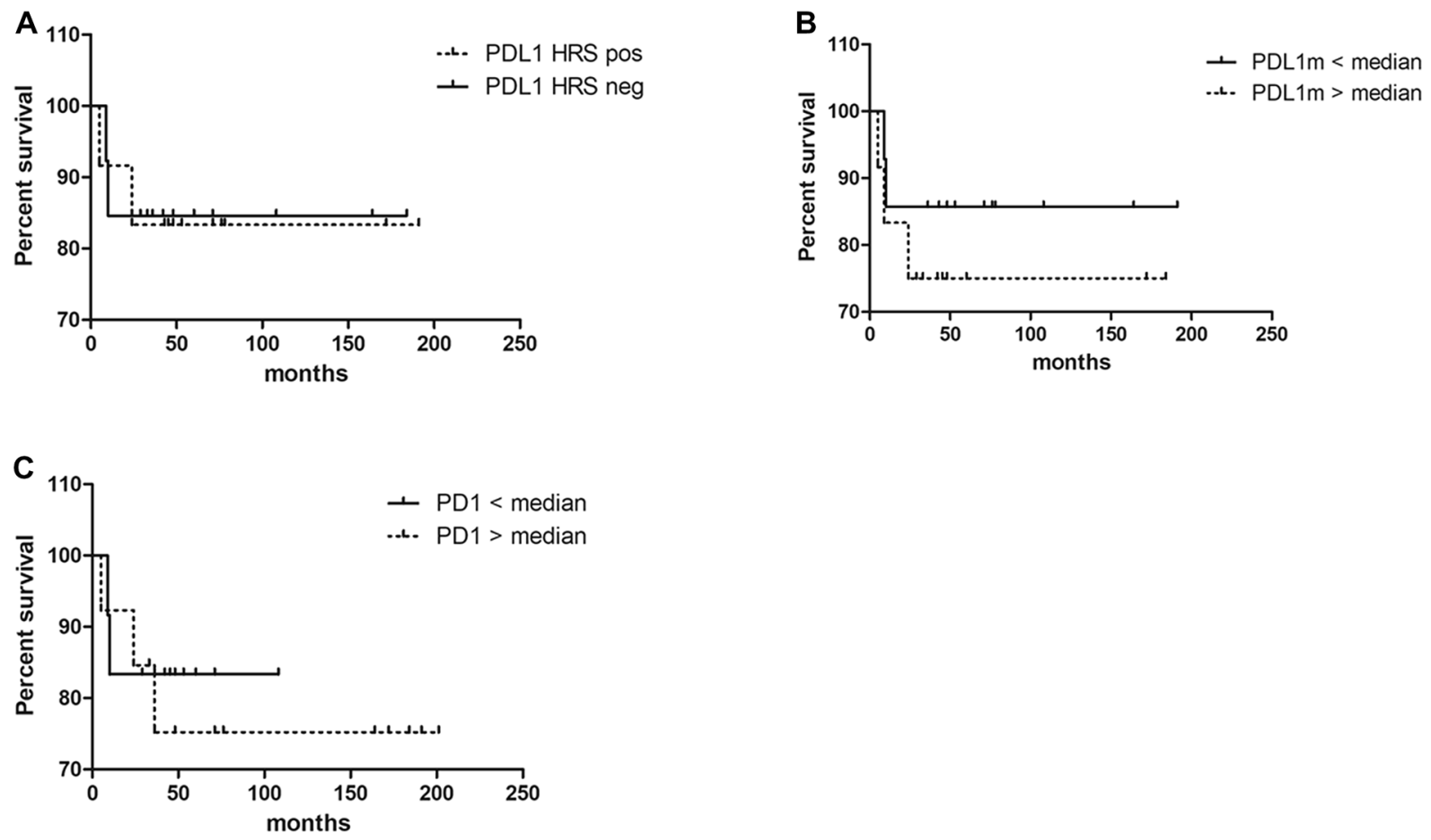

Fig. 3 Event-free survival (EFS) analysis to PDL1+HRS cells (a), PDL1+cells at microenvironment (cut off 50th percentile) (b) and PD1 + cells at the microenvironment (cut off 50th percentile) (c)

studied PD1/PDL1 in cHL by means of IHC, who found a low PD1 expression in $<20 \%$ of cases, whereas PDL1 in HRS tumor cells was observed in all cases [33]. Conversely, in our series, PDL1 expression in HRS cells was proved only in $44 \%$ of cases. Furthermore, amplification, copy gain and both were observed in $8 \%, 16 \%$ and $14 \%$ of cases, respectively, indicating that PDL1 expression and genetic alteration did not play a key role in lymphomagenesis in the cHL cases analyzed. In line with this, PDL1 expression was not associated with event-free survival, while outcome could not be analyzed in relation to PDL1 genetic alterations given that this analysis only included 37 cases.

A tolerogenic environment characterized by PD1 expression was proved in DLBCL [34], in particular in GC-type [16]. In line with this, PD1 levels are increased in tumorinfiltrating T cells in cHL compared to healthy volunteers, even in peripheral blood [35]. The increased PD1 + in tumor-infiltrating lymphocytes was consistently associated with poor prognosis in cHL patients treated with conventional therapies [10]. Conversely, no effect of PD1 + cells on tumor-infiltrating lymphocytes was observed on survival in our pediatric $\mathrm{cHL}$ when the median number of PD1 + cells was used as the cutoff for survival analysis. Our group previously demonstrated that EBV was statistically associated with children younger than 10 years old in several types of lymphomas [23], that also include pediatric cHL previously studied that integrate part of this analysis [22, 27], then providing the bases for EBV and age groups evaluation. EBV presence was associated with increased cytotoxic effector cells at the microenvironment in DLBCL, in coexistence with the PD1 + tolerogenic milieu [34], whereas this cytotoxic profile was proved in $\mathrm{cHL}$ in children younger than 10 years in cHL [27]. The immunomodulatory role of IL10 in EBV-associated lymphomas, as well as in EBV carriers, was previously demonstrated, suggesting a viral-induced balance between cytotoxic and immunosuppressive features [34, 36]. Moreover, IL10 upregulation by LMP1 viral protein was demonstrated in vitro [37]. In addition, HRS and Tregs cells in the microenvironment secrete soluble factors, such as IL10 and TGF $\beta$, to induce an immunoregulatory milieu, which includes enhanced PD1 signaling [38]. Cooperation between IL10 and PD1 was also demonstrated in vitro, given that both IL10 and PD1 blockade enhance the activity of CD8 + T cell in melanoma patients [39]. Therefore, the significant correlation observed between PD1 + and IL10 + cells may reflect a cooperative immunoregulatory role in EBV + cases and in children younger than 10 years old, possibly to counteract viral-induced cytotoxicity. In contrast, in EBV - cases, since IL10 is not induced by EBV presence, TGF $\beta$ may cooperate with PD1 to achieve immune modulation at the microenvironment.

EBV presence, through LMP1 protein, induces PDL1 expression, suggesting an alternative mechanism for PDL1 induction in cHLs with diploid 9p24.1 [17], while 
a counter-regulatory role for EBV miR-BHRF1-2-5p in vitro was proved to balance PDL1 surface protein expression [40]. PDL1 expression was associated with EBV expressing latency II or III patterns in post-transplant lymphoproliferative disorder (PTLD) [41], and a high frequency of PDL1 genetic aberrations was observed in several types of EBV-associated lymphomas [18]. In our series, even though LMP1 expression was proved in all pediatric EBV + cHL, no increase in genetic aberrations was observed in EBV-associated cases. Furthermore, concerning PDL1 expression, neither PDL1 positivity nor mean cell count in PDL1 + HRS cells were observed when $\mathrm{EBV}+$ cases were compared with EBV - ones. This finding might reveal that, unlike adult lymphomas [18, 41], EBV presence as well as the expression of its major oncogenic protein, LMP1, has no effect on PDL1 gene or protein expression in pediatric cHL. When PDL1 expression was discriminated between tumor and microenvironment immune cells, PDL1 + cells from both were significantly associated with EBV presence in adult DLBCL [16]. In our series, PDL1 expression was increased in EBV-associated pediatric $\mathrm{cHL}$ cases exclusively at the microenvironment. The presence of regionally localized PDL1 + macrophages that form a micro-environmental niche for HRS cells in adult cHL was suggested [14]. Therefore, our results may indicate that this PDL1 + niche in pediatric cHL could be observed only in the context of EBV-associated cases, in a population with high incidence of pediatric EBV infection [23].

In summary, our results reveal that, even though a high cytotoxic environment was described in EBV-related pediatric cHL [25, 27], it might be counterbalanced by an immunoregulatory cooperation between of PD1 and IL10, along with a micro-environmental PDL1 + niche. This regulation may render a cytotoxic microenvironment that unsuccessfully try to eliminate EBV + HRS tumor cells in pediatric patients.

Acknowledgements The authors thank Barbara Cao; Silvana Romero; Cristina Pabes and Maria Jose Andrade (Histopathological Laboratory, at the Ricardo Gutierrez Children's Hospital) for their helpful histotechnical work.

Author contributions $\mathrm{CP}$ conceived of the study and its design, drafted the manuscript and acquired funding. JO and CS performed the experiments, the analysis and interpretation of data. GLM, DME and PMV participated in its coordination and modification. All authors read and approved the final manuscript.

Funding This study was supported in part by grants from National Agency for Science and Technology Promotion (PICT $2015 \mathrm{n}^{\circ} 1533$ PID clinic $n^{\circ} 048$ - PICT 2017 1554). CP, DME and PMV are members of the CONICET Research Career Program. JO is a CONICET doctoral fellow.
Data availability The datasets used and/or analysed during the current study are available from the corresponding author on reasonable request.

\section{Compliance with ethical standards}

Conflict of interest The authors declare that they have no competing interests.

Ethical approval and consent to participate Institutional guidelines regarding human experimentation were followed, according to the Helsinki Declaration of 1975. The protocol was approved by the Ethical Committee of Ricardo Gutierrez Children Hospital, and written informed assent and consent was obtained from all patients or patient's parents depending on age.

\section{References}

1. Liu Y, Sattarzadeh A, Diepstra A, Visser L, van den Berg A (2014) The microenvironment in classical Hodgkin lymphoma: an actively shaped and essential tumor component. Semin Cancer Biol 24:15-22

2. Seifert M, Scholtysik R, Küppers R (2019) Origin and pathogenesis of B cell lymphomas. Methods Mol Biol 1956:1-33

3. Hanahan D, Weinberg RA (2011) Hallmarks of cancer: the next generation. Cell 144:646-674

4. Wherry EJ, Ahmed R (2004) Memory CD8 T-cell differentiation during viral infection. J Virol 78:5535-5545

5. Jiang Y, Li Y, Zhu B (2015) T-cell exhaustion in the tumor microenvironment. Cell Death Dis 6:e1792

6. Bryan LJ, Gordon LI (2015) Blocking tumor escape in hematologic malignancies: the anti-PD-1 strategy. Blood Rev 29:25-32

7. Küppers R, Engert A, Hansmann ML (2012) Hodgkin lymphoma. J Clin Invest 122:3439-3447

8. Tzankov A, Meier C, Hirschmann P, Went P, Pileri SA, Dirnhofer S (2008) Correlation of high numbers of intratumoral FOXP3+ regulatory $\mathrm{T}$ cells with improved survival in germinal center-like diffuse large B-cell lymphoma, follicular lymphoma and classical Hodgkin's lymphoma. Haematologica 93:193-200

9. Carreras J, Lopez-Guillermo A, Roncador G, Villamor N, Colomo L, Martinez A, Hamoudi R, Howat WJ, Montserrat E, Campo E (2009) High numbers of tumor-infiltrating programmed cell death 1-positive regulatory lymphocytes are associated with improved overall survival in follicular lymphoma. J Clin Oncol 27:1470-1476

10. Muenst S, Hoeller S, Dirnhofer S, Tzankov A (2009) Increased programmed death-1+ tumor-infiltrating lymphocytes in classical Hodgkin lymphoma substantiate reduced overall survival. Hum Pathol 40:1715-1722

11. Vardhana S, Younes A (2016) The immune microenvironment in Hodgkin lymphoma: T cells, B cells, and immune checkpoints. Haematologica 101:794-802

12. Roemer MG, Advani RH, Ligon AH, Natkunam Y, Redd RA, Homer H, Connelly CF, Sun HH, Daadi SE, Freeman GJ, Armand P, Chapuy B, de Jong D, Hoppe RT, Neuberg DS, Rodig SJ, Shipp MA (2016) PD-L1 and PD-L2 genetic alterations define classical Hodgkin lymphoma and predict outcome. J Clin Oncol 34:2690-2697

13. Keir ME, Butte MJ, Freeman GJ, Sharpe AH (2008) PD-1 and its ligands in tolerance and immunity. Annu Rev Immunol 26:677-704 
14. Carey CD, Gusenleitner D, Lipschitz M, Roemer MGM, Stack EC, Gjini E, Hu X, Redd R, Freeman GJ, Neuberg D, Hodi FS, Liu XS, Shipp MA, Rodig SJ (2017) Topological analysis reveals a PDL1-associated microenvironmental niche for Reed-Sternberg cells in Hodgkin lymphoma. Blood 130:2420-2430

15. Patel SS, Weirather JL, Lipschitz M, Lako A, Chen PH, Griffin GK, Armand P, Shipp MA, Rodig SJ (2019) The microenvironmental niche in classic Hodgkin lymphoma is enriched for CTLA-4-positive T cells that are PD-1-negative. Blood 134:2059-2069

16. Kiyasu J, Miyoshi H, Hirata A, Arakawa F, Ichikawa A, Niino D, Sugita Y, Yufu Y, Choi I, Abe Y, Uike N, Nagafuji K, Okamura T, Akashi K, Takayanagi R, Shiratsuchi M, Ohshima K (2015) Expression of programmed cell death ligand 1 is associated with poor overall survival in patients with diffuse large B-cell lymphoma. Blood 126:2193-2201

17. Green MR, Rodig S, Juszczynski P, Ouyang J, Sinha P, O’Donnell E, Neuberg D, Shipp MA (2012) Constitutive AP-1 activity and EBV infection induce PD-L1 in Hodgkin lymphomas and posttransplant lymphoproliferative disorders: implications for targeted therapy. Clin Cancer Res 18:1611-1618

18. Kataoka K, Miyoshi H, Sakata S, Dobashi A, Couronné L, Kogure Y, Sato Y, Nishida K, Gion Y, Shiraishi Y, Tanaka H, Chiba K, Watatani Y, Kakiuchi N, Shiozawa Y, Yoshizato T, Yoshida K, Makishima H, Sanada M, Onozawa M, Teshima T, Yoshiki Y, Ishida T, Suzuki K, Shimada K, Tomita A, Kato M, Ota Y, Izutsu K, Demachi-Okamura A, Akatsuka Y, Miyano S, Yoshino T, Gaulard P, Hermine O, Takeuchi K, Ohshima K, Ogawa S (2019) Frequent structural variations involving programmed death ligands in EpsteinBarr virus-associated lymphomas. Leukemia 33:1687-1699

19. Saito R, Abe H, Kunita A, Yamashita H, Seto Y, Fukayama M (2017) Overexpression and gene amplification of PD-L1 in cancer cells and PD-L1+ immune cells in Epstein-Barr virus-associated gastric cancer: the prognostic implications. Mod Pathol 30:427-439

20. Shannon-Lowe C, Rickinson A (2019) The global landscape of EBV-associated tumors. Front Oncol 9:713

21. Shanbhag S, Ambinder RF (2018) Hodgkin lymphoma: a review and update on recent progress. CA Cancer J Clin 68:116-132

22. De Matteo E, García Lombardi M, Preciado MV, Chabay P (2019) Changes in EBV association pattern in pediatric classic Hodgkin lymphoma from a single institution in argentina. Front Oncol 9:881

23. Chabay PA, Preciado MV (2013) EBV primary infection in childhood and its relation to B-cell lymphoma development: a minireview from a developing region. Int J Cancer 133:1286-1292

24. Tan GW, Visser L, Tan LP, van den Berg A, Diepstra A (2018) The microenvironment in epstein-barr virus-associated malignancies. Pathogens 7:E40

25. Barros MH, Vera-Lozada G, Soares FA, Niedobitek G, Hassan R (2012) Tumor microenvironment composition in pediatric classical Hodgkin lymphoma is modulated by age and Epstein-Barr virus infection. Int J Cancer 131:1142-1152

26. Barros MH, Segges P, Vera-Lozada G, Hassan R, Niedobitek G (2015) Macrophage polarization reflects T cell composition of tumor microenvironment in pediatric classical Hodgkin lymphoma and has impact on survival. PLoS ONE 10:e0124531

27. Jimenez O, Barros MH, De Matteo E, Garcia Lombardi M, Preciado MV, Niedobitek G, Chabay P (2019) M1-like macrophage polarization prevails in young children with classic Hodgkin Lymphoma from Argentina. Sci Rep 9:12687

28. Swerdlow SH, Campo E, Pileri SA, Harris NL, Stein H, Siebert R, Advani R, Ghielmini M, Salles GA, Zelenetz AD, Jaffe ES (2016) The 2016 revision of the World Health Organization classification of lymphoid neoplasms. Blood 127:2375-2390

29. Juneja VR, McGuire KA, Manguso RT, LaFleur MW, Collins N, Haining WN, Freeman GJ, Sharpe AH (2017) PD-L1 on tumor cells is sufficient for immune evasion in immunogenic tumors and inhibits CD8 T cell cytotoxicity. J Exp Med 214:895-904
30. Li L, Sun R, Miao Y, Tran T, Adams L, Roscoe N, Xu B, Manyam G, Tan X, Zhang H, Xiao M, Tzankov A, Visco C, Dybkaer K, Bhagat G, Tam W, Hsi E, van Krieken J, You H, Huh J, Ponzoni M, Ferreri A, Moller M, Piris M, Zhang M, Winter J, Medeiros L, Rassidakis G, Vaupel C, Li Y, Dakappagari N, Xu-Monette Z, Young K (2019) PD-1/PD-L1 expression and interaction by automated quantitative immunofluorescent analysis show adverse prognostic impact in patients with diffuse large B-cell lymphoma having T-cell infiltration: a study from the International DLBCL Consortium Program. Mod Pathol 32:741-754

31. Li-Yang Hu, Xiao-Lu Xu, Rao H-L, Chen J, Lai R-C, Huang H-Q, Jiang W-Q, Lin T-Y, Xia Z-J, Cai Q-Q (2017) Expression and clinical value of programmed cell death-ligand 1 (PD-L1) in diffuse large B cell lymphoma: a retrospective study. Chin J Cancer 36:94

32. Wang Y, Wenzl K, Manske M, Asmann Y, Sarangi V, Greipp P, Krull J, Hartert K, He R, Feldman A, Maurer M, Slager S, Nowakowski G, Habermann T, Witzig T, Link B, Ansell S, Cerhan J, Novak A (2019) Amplification of 9p24.1 in diffuse large B-cell lymphoma identifies a unique subset of cases that resemble primary mediastinal large B-cell lymphoma. Blood Cancer J 9:73

33. Dilly-Feldis M, Aladjidi N, Refait J, Parrens M, Ducassou S, Rullier A (2019) Expression of PD-1/PD-L1 in children's classical Hodgkin lymphomas. Pediatr Blood Cancer 66:e27571

34. Cohen M, Vistarop AG, Huaman F, Narbaitz M, Metrebian F, De Matteo E, Preciado MV, Chabay PA (2017) Cytotoxic response against Epstein Barr virus coexists with diffuse large B-cell lymphoma tolerogenic microenvironment: clinical features and survival impact. Sci Rep 7:10813

35. Yamamoto R, Nishikori M, Kitawaki T, Sakai T, Hishizawa M, Tashima M, Kondo T, Ohmori K, Kurata M, Hayashi T, Uchiyama T (2008) PD-1-PD-1 ligand interaction contributes to immunosuppressive microenvironment of Hodgkin lymphoma. Blood 111:3220-3224

36. Vistarop AG, Cohen M, Huaman F, Irazu L, Rodriguez M, De Matteo E, Preciado MV, Chabay PA (2018) The interplay between local immune response and Epstein-Barr virus-infected tonsillar cells could lead to viral infection control. Med Microbiol Immunol 207:319-327

37. Lambert S, Martinez O (2007) Latent membrane protein 1 of EBV activates phosphatidylinositol 3-kinase to induce production of IL-10. J Immunol 179:8225-8234

38. Liu W, Shipp M (2017) Signaling pathways and immune evasion mechanisms in classical Hodgkin lymphoma. Blood 130:2265-2270

39. Sun Z, Fourcade J, Pagliano O, Chauvin JM, Sander C, Kirkwood JM, Zarour HM (2015) IL10 and PD-1 cooperate to limit the activity of tumor-specific CD8+ T cells. Cancer Res 75:1635-1644

40. Cristino A, Nourse J, West R, Sabdia M, Law S, Gunawardana J, Vari F, Mujaj S, Thillaiyampalam G, Snell C, Gough M, Keane C, Gandhi M (2019) EBV microRNA-BHRF1-2-5p Targets the 3'UTR of immune checkpoint ligands PD-L1 and PD-L2. Blood 134:2261-2270

41. Veloza L, Teixido C, Castrejon N, Climent F, Carrió A, Marginet M, Soldini D, González-Farré B, Ribera-Cortada I, Lopez-Guillermo A, González-Barca C, Sierra A, Herrera M, Gómez C, Garcia A, Balagué O, Campo E, Martinez A (2019) Clinicopathological evaluation of the programmed cell death 1 (PD1)/programmed cell death-ligand 1 (PD-L1) axis in post-transplant lymphoproliferative disorders: association with epstein-barr virus, PD-L1 copy number alterations, and outcome. Histopathology 75:799-812

Publisher's Note Springer Nature remains neutral with regard to jurisdictional claims in published maps and institutional affiliations. 